\title{
High-Order Thinking Skills: The Educational Treasure Hunt Game
}

Yogi Udjaja ${ }^{1, *}$, Sasmoko², Jurike V. Moniaga ${ }^{1,}$ Kevin Zulfian Bay ${ }^{1}$

${ }^{1}$ Computer Science Department, School of Computer Science, Bina Nusantara University, Jakarta, 11480, Indonesia

${ }^{2}$ Primary Teacher Education Department, Faculty of Humanities, Bina Nusantara University, Jakarta, 11480, Indonesia

\begin{tabular}{l} 
A R T I C L E I N F O \\
\hline Article history: \\
Received: 27 March, 2020 \\
Accepted: 12 May, 2020 \\
Online: 22 July, 2020 \\
\hline Keywords: \\
HOTS Game \\
Educational Video Games \\
Treasure Hunt \\
Puzzle \\
Game Development \\
\hline
\end{tabular}

\begin{abstract}
A B S T R A C T
Many people who have no experience want to make Games. Among them are people who only like to play Games, people who want to make business through Games, and there are also people who really want to make Games. Assume making the game is very difficult and confusing, but forget the many examples that already exist, and can evolve if modified only slightly. In this case, there are several genres of games that are quite popular, which can be changed and modified into another sub-genre. One of them is the Treasure Hunt. It is one of the well-known genres in various video games and has a straight and simple design that is also easy to understand. Which is used in a game that aims to "Find and Work".
\end{abstract}

\section{Introduction}

Lack of perseverance and one's desire when pushed to work on a problem is very common among people. Especially if the individual is required to work on mathematical-themed problems. Indeed, when you're relaxed, people are lazy to do something, especially doing something that in general, or almost everyone, hates it.

There are several methods that can be done to encourage someone's desire or willingness to do and work on these problems. And some of them have their own different interests. A very effective way, at least in this digital age.

Which entertainment and media can be easily obtained and enjoyed only with a smartphone and one of them is video games, which are almost from all walks of life, and at least have played, really enjoyed video games and a genre that is very popular among young and old alike, namely A Series of Clues. Or rather Puzzle and Treasure Hunt [1].

And There is High Order Thinking Skills (HOTS). The game that has both Treasure Hunt and an Adventure Puzzle genre that aims to represent the $3 \mathrm{~T}$ regions (Leading, Outermost, and

${ }^{*}$ Yogi Udjaja, Jakarta, Indonesia 11480, +62 898268 3399,

udjaja.yogi@gmail.com, yogi.udjaja@binus.ac.id

www.astesj.com

https://dx.doi.org/10.25046/aj050424
Disadvantaged) in Indonesia and train the ability to think through puzzle games [2].

HOTS Game was developed for mobile devices with the Android platform which will be easily accessible to most people [3].

HOTS Game is a game with adventure and puzzles in it. The puzzles in the game are math problems that contain some Creativity, Critical Thinking, and Problem Solving. The target players of the game are for all people but the main goal is elementary school children with the aim of training complex thinking skills. Not only that, but also aimed at encouraging the strength of one's thinking and one's skills at a large age.

Follow the story line starting from one point to another point. Get a few clues to interpret, then examine it for later to be used as a pointer. Look for a series of continuous problems. which are hidden somewhere in areas located in Indonesia.

Accompanied by music and an atmosphere that focuses on important points in the regions of Indonesia, to add to the impression of culture and nationality.

By covering various aspects given from most Video Games, such as Atmosphere, Music, Storyline, puzzles, etc. Where will it add to one's desire to get things done. Completion or game 


\section{Y. Udjaja et al. / Advances in Science, Technology and Engineering Systems Journal Vol. 5, No. 4, 196-202 (2020)}

observation where completing all missions, getting all achievements and collectible is also the biggest motivation that is most liked. There is also Fantasy, where they prefer games where they can feel like someone else in another place. And the third is Design [4].

\section{Related Research}

\subsection{Video Game}

Is a type of play activity, which is carried out in the context of mock realities, where participants try to achieve predetermined goals and mediate in the form of digital media [5]. To play a game, one must play it on the right platform. Platform games can be categorized into three types: console, cellular, and cross-platform. Each platform has different SDK distribution characteristics and methods. The console SDK has a closed distribution method, while most mobile SDKs can be downloaded freely, although with some constraints. That is why the development of mobile games is becoming increasingly popular [6].

\subsection{Treasure Hunt}

A treasure hunt type game that uses positioning. Players are given clues or directions to proceed along with predetermined treasure hunt routes based on their location as determined by the clues. Based on these clues or directions, players change their locations. Based on these changed locations, as determined by the GPS, additional clues or directions are given to the players until one of the players completes the treasure hunt routes and arrives at the treasure to win the game. The game also incorporates other variables in determining the clues to provide to players, such as the location of other players as determined by their GPS devices, the previous locations of the players and other players and whether the player has correctly solved certain clues [7].

\subsection{User Interface}

An Interface Experience is information that is intended between the Player and the Device he uses. In Video Games, the Interface takes the form of a Controller, and much more to the Graphical User Interface.

As the name implies, the GUI contains Graphics-Visual information. With the lack of physical form and pleasantness, relying on Display to send information to players. The quality of the UI can be assessed from two aspects [8,9]:

1. Usability: How well it facilitates the intended purpose of the tool, and how easy it is for users to absorb and operate.

2. Consistency: performing the same actions several times will produce the same results. However, that does not mean that consistently bad results are good.

\subsection{User Experience}

Different, but directly related to UI, User Experience concerns the quality of the user's time with the product [3, 10-12], which can certainly affect the UI. Where the UI is about how well a tool can convey the information it needs to users, UX is about how fun it is to use it. The interface can be very bad in providing all important information, but users might still consider it a good tool, for several reasons such as ease of use and aesthetically pleasing design.

\section{Research Method}

Like the Game Development Life Cycle [13-15], In the Initiation Phase, some problems have been thought by people to be lacking in the desire to do something. So, made a game that is intended to encourage the spirit of someone to do it. Which at least makes the individual want to try.

In the next Phase, Game System has been analyzed, and made a design. How this game will run and focus to help players increase their desires and also made in writing.

In the production phase, the Designer makes some basic assets needed to just try. Creating several data sets as test data such as character names and scores. Make a number of basic programming sets to focus the player looking for a hidden problem [16].

In the alpha testing phase, adjustments are made to the Design Document about the game. And do some bug testing and how the game runs. Collect some basic player data such as username and score and evaluate it.

Then in the Beta Testing phase, a number of surveys are conducted to the players which are focused on how the players react to how to find a set of puzzles and do the questions that have been made. A written test is also carried out.

Some feedback from players who have been tested, mostly including the difficulty of the questions presented. But this problem is ignored, because this game is intended to give ordinary people who are in the public area, a problem with a fairly high level

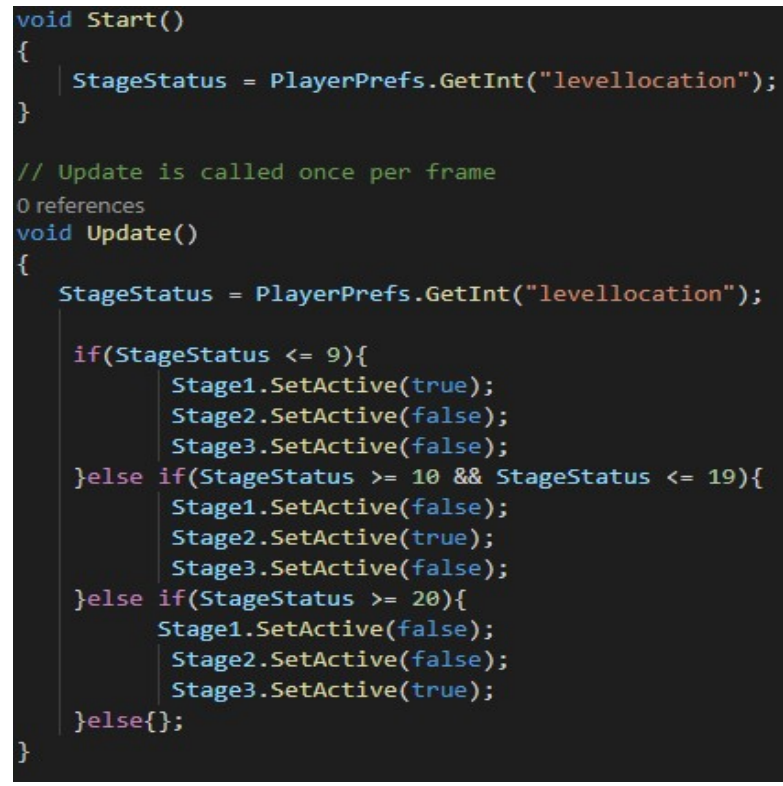

Figure 1: Unlock Method 


\section{Y. Udjaja et al. / Advances in Science, Technology and Engineering Systems Journal Vol. 5, No. 4, 196-202 (2020)}

Almost all the methods used are basic and have logic that is easy to apply to anyone. Although many seem to be able to give a faster or more efficient effect, but here are some methods that use a little extra that can add effectiveness.

In the last phase, the release was being held for some times, then proceeded to release it via Google Play Store and also still do some repairs and maintenance at time.

\subsection{Unlocks}

In this game, many use the method that is locked and must be obtained. Use something simple, just by validating the status of the player's progression, and making it active.

\subsection{Transition}

Transition from one view to another, using the SceneManager which is divided into several functions and methods. And will be called in each particular situation.

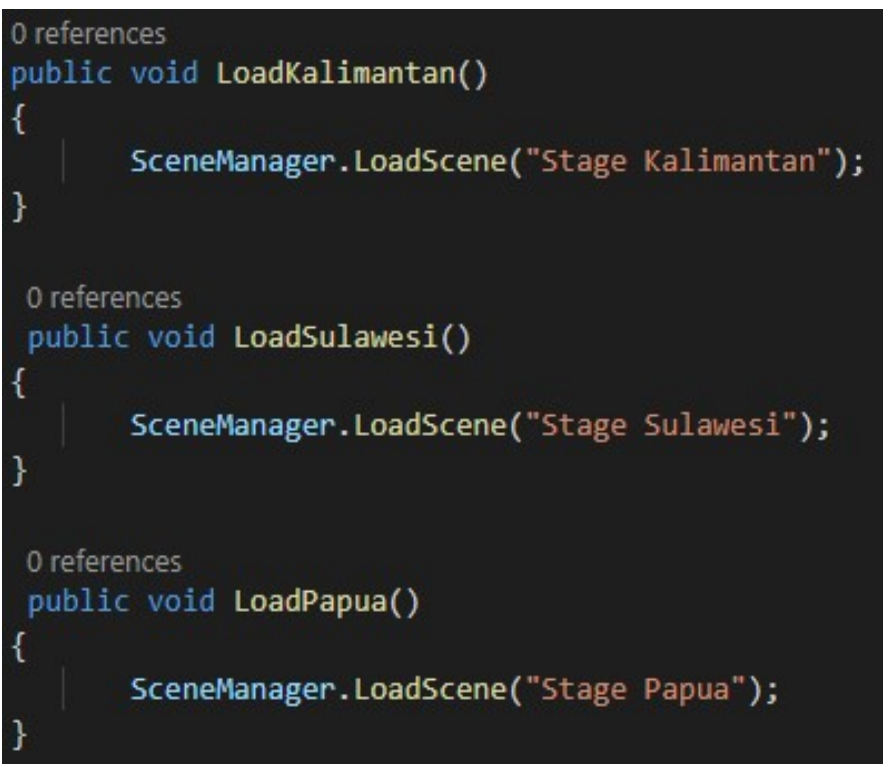

Figure 2: Loading Stages

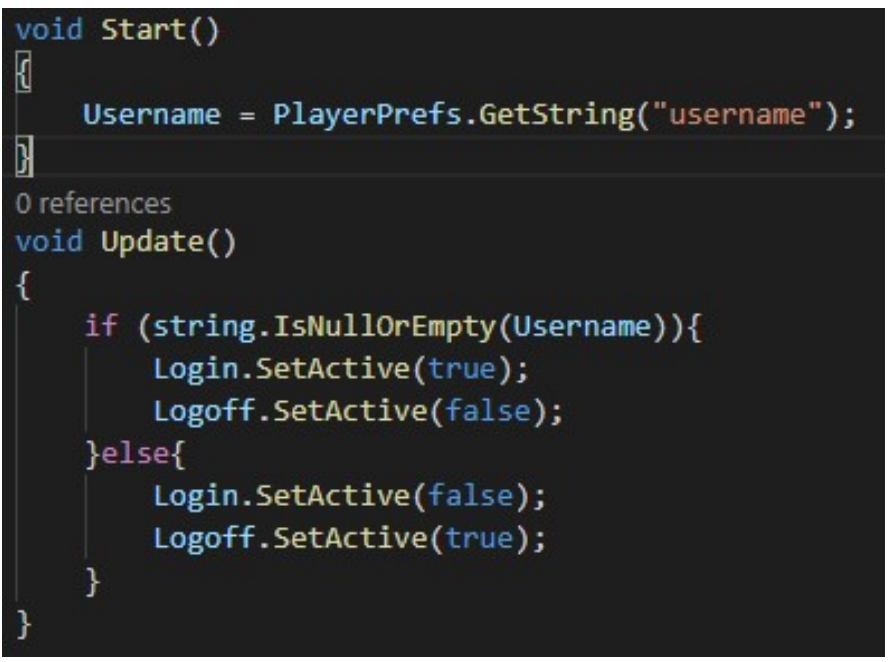

Figure 3: Logged Status
And in some cases, as in the Title Screen, only use two different canvases, that is, the display status Logged-In, and the status Logged-Off.

\subsection{Data Management}

For storing data and status obtained by the player from the results of solving the problem, as well as from the input requested in each game, as well as data taken from the value of an object, the Get Playerpref function is used.

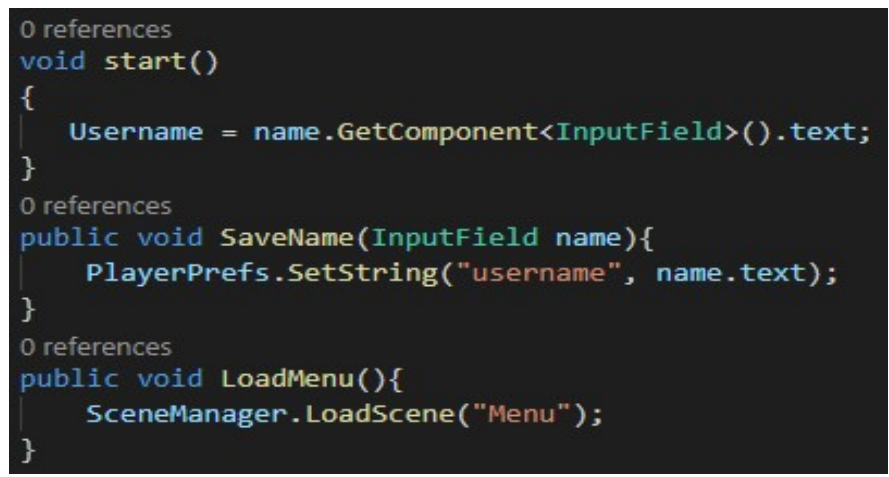

Figure 4: Setting Data

And to send and specify the desired data. Get Playerpref function will be used. The function is used in several different uses and different data types.

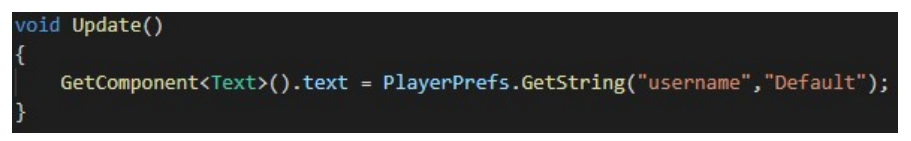

Figure 5: Loading Username

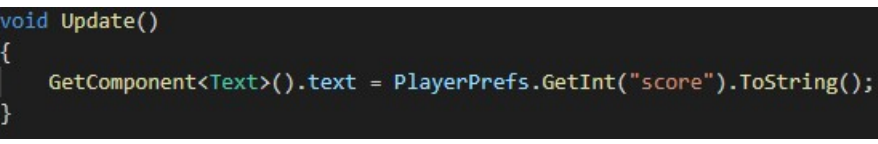

Figure 6: Loading Score

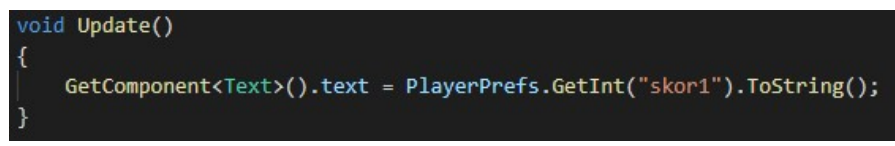

Figure 7: Loading Specific Score

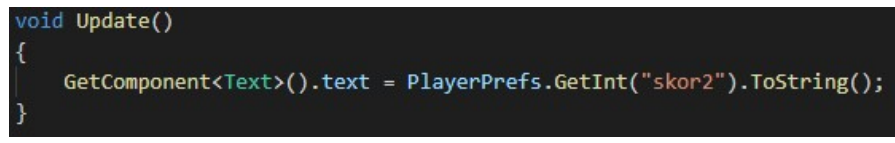

Figure 8: Loading Specific Score 2

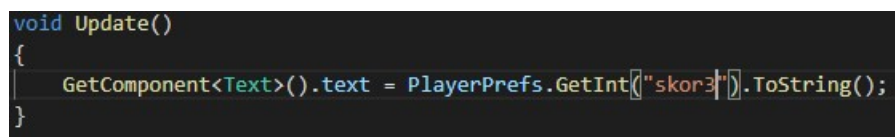

Figure 9: Loading Specific Score 3

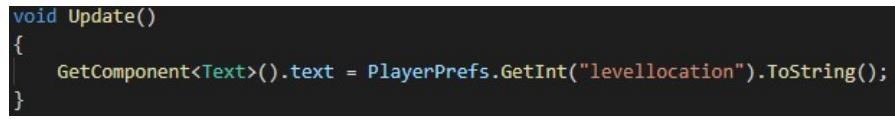

Figure 10: Loading Location Status 


\section{Analisys}

We conducted a survey by giving 5 mathematical-themed problems, in writing. And gave the same problem again, but required to do it using a video game. Of the 30 people we surveyed, two statistics were obtained.

\subsection{Total questions answered}

From 30 that has been surveyed, there are 2 people that can answer 1 Question and only 1 that can answer 2 Questions. Then the rest can't answer at all.

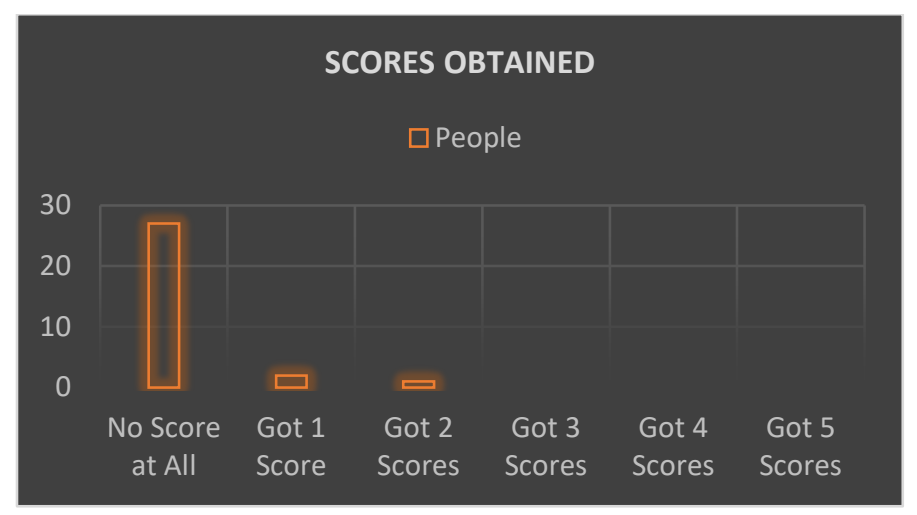

Figure 11: Total Answered Pre-Test

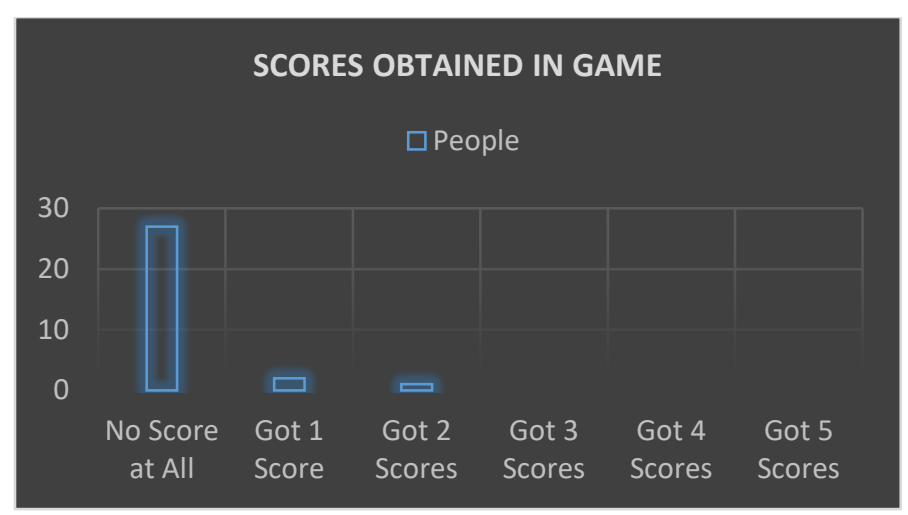

Figure 12: Total Answered Post Test (in-game)

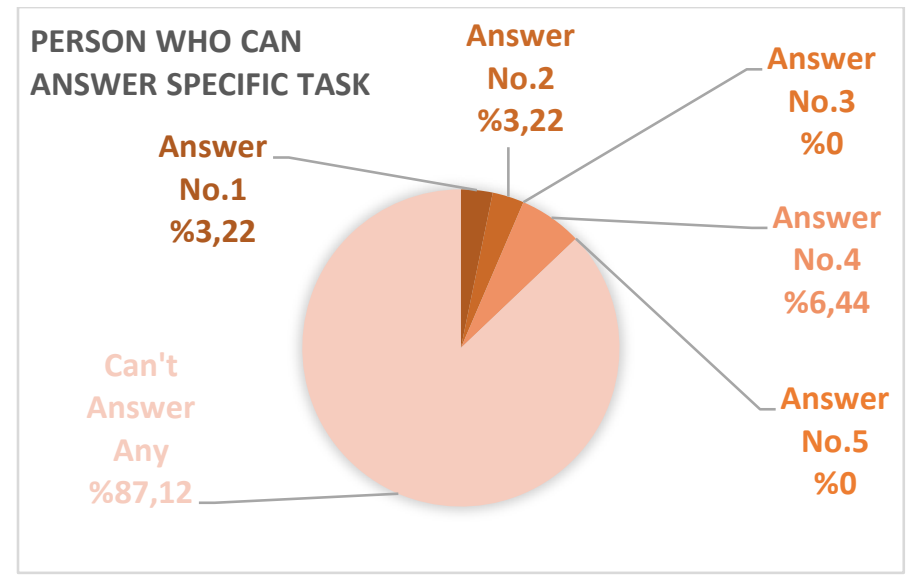

Figure 13: Task Answered

\subsection{Specific Number Answered}

Only one person can answer Problem number 1, also one person can answer Problem number 2 and that one person earlier that can answer Problem number 2, can also answer Problem number 4 , whereas no one can answer questions number 3 and number 5 .

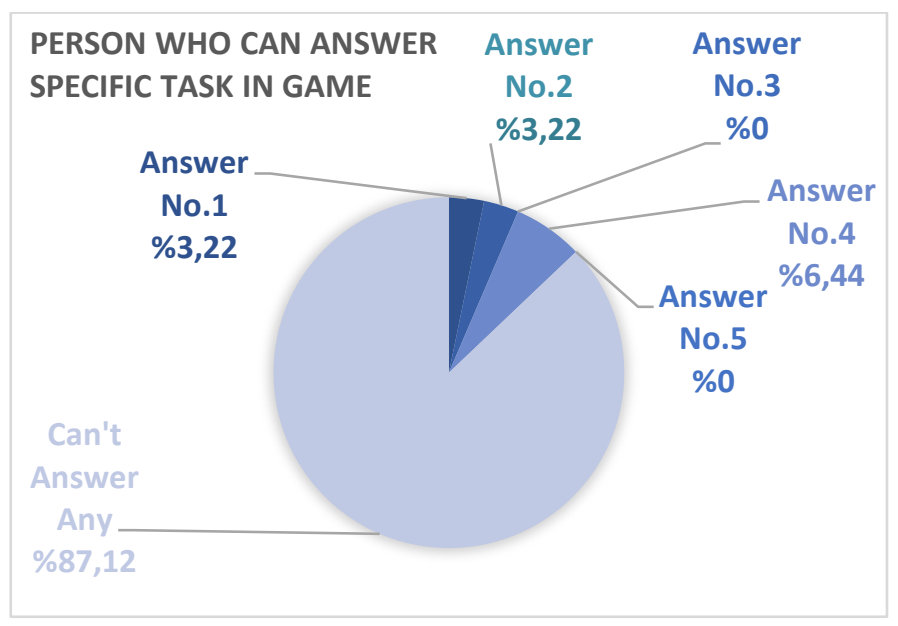

Figure 14: Task Answered in-game

\subsection{Difference}

Among those who can answer their questions in writing, they will easily answer the questions in the Game. Yes, it is because the working period from written to game is quite short.

But there are no changes that occur to people who are unable to answer written questions, then to questions on the Game.

Assuming the difficulty of finding people, who are happy to work on math-related problems seriously in their spare time. Where most people tend to be reluctant to deal with things that require them to think hard when being relaxed.

\section{Result and Discussion}

\subsection{Storyline}

Indonesia is a beautiful country and rich in culture. The richness of Indonesian culture comes from the legacy of ancestors who always passed down to their future generations. Certainly, an ancestral inheritance has a very valuable heritage to guard. It is the duty of each region to safeguard the cultural treasures they possess. Saipul, an adventurous child, loves Indonesia very much and the cultural riches it possesses. Saipul is always passionate about knowing new cultures that have not been exposed to the outside world. Currently there are still 3 areas that he wants to explore. Namely East Kalimantan, South Sulawesi and West Papua. Saipul wants to know what kind of heritage the three regions have. To explore these areas, Saipul will be faced with a puzzle challenge that will direct him to the relics of each region. To achieve this, Saipul must complete the puzzle quickly and precisely to get clues to the next puzzle. 


\subsection{Title Screen}

There are two types of Title Screen in this game, the first is the Normal version, and the other one is the Logged-In version. On the Title Screen the Normal player version will be shown the Game Logo, the Background depicts the Indonesian Archipelago, Input Field for Username and Button to login.

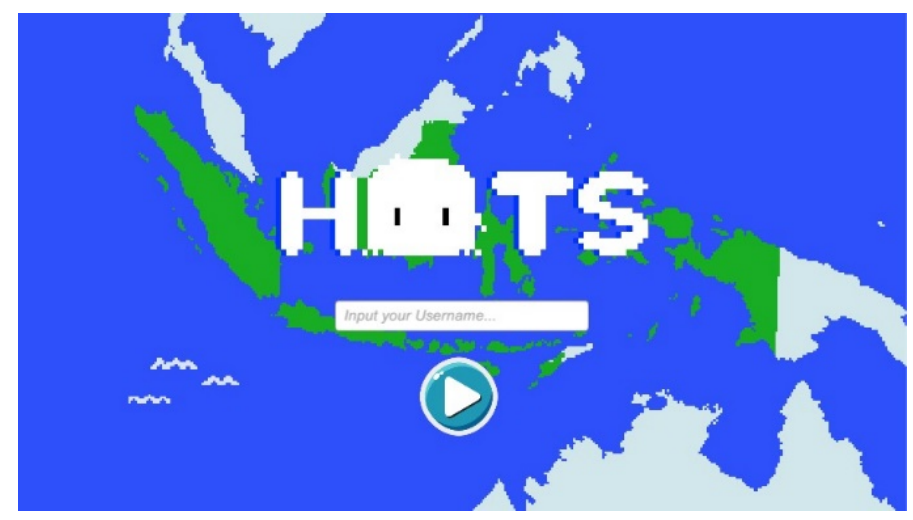

Figure 15: Title Screen

If the Player has filled in the Username and has already logged in, the Title Screen display will change to Logged-In. Then the Title Screen will display the phrase "Welcome Back" along with the Player Username. Two new buttons will also be displayed, namely the "Play" and "Exit" The "Play" button is used to proceed to the next Scene, the Main Menu. While the "Exit" button is used to exit the game, as well as log out from the player's username.

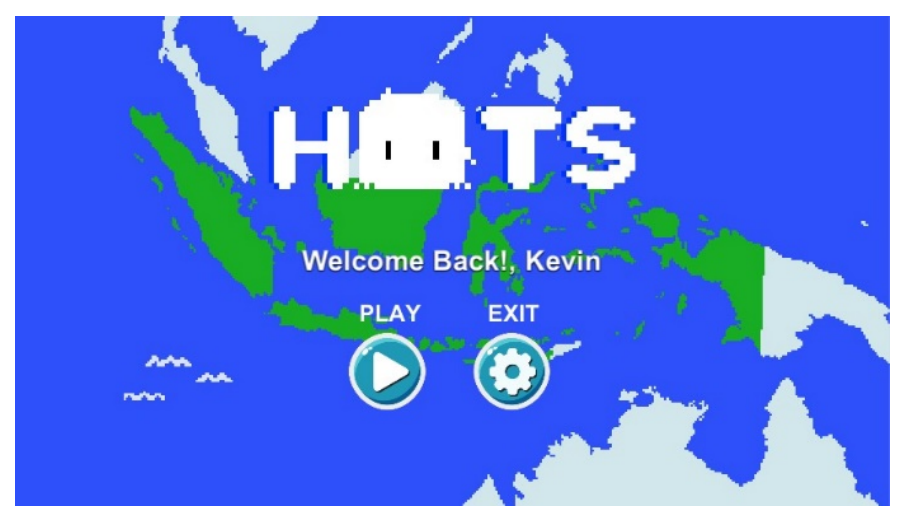

Figure 16: Alternate Title Screen

\subsection{Main Menu}

On the main menu, players will be shown a Map of Indonesian Island, which has a yellow area, which indicates that there are stages that can be played on the island. However, not all the available Stages are available at the start of the Game. The Stages are still locked and will open if the player has performed certain conditions. The playable stage will be indicated by the Exclamation Mark symbol in Orange. Each symbol of the Stage is also accompanied by a Panel containing the name of the Stage or Island and a Description presented by the Stage.

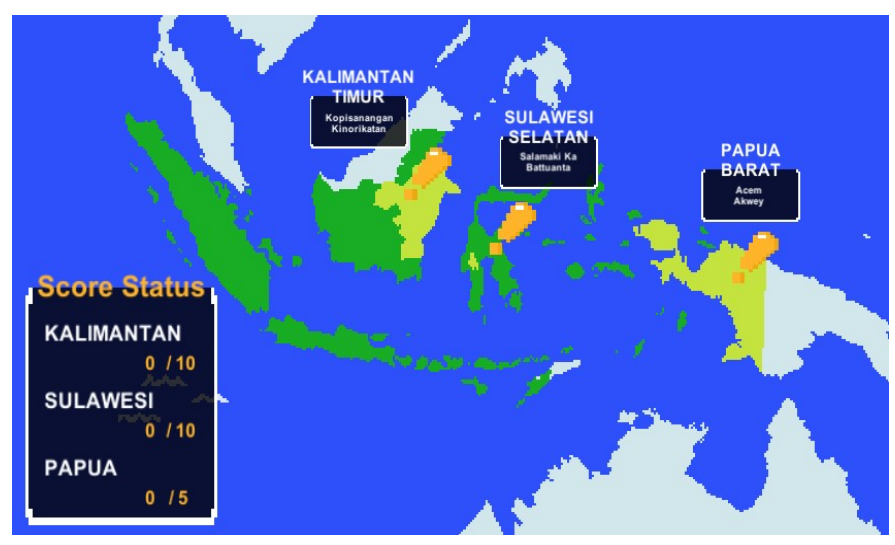

Figure 17: Stage Menu

Then there is also a panel at the bottom left of the Screen, called the Score Status Panel. This panel aims to show the Progress or Status Score that the player has obtained from each question on the existing Stages. The Panel has three indications that show the Player's Score and Total Score can be obtained from each Stage.

\subsection{Gameplay}

As both Treasure Hunt and Puzzle game HOTS Game has a fast and precise puzzle-solving gameplay. To proceed to the next puzzle, the player must complete it perfectly, that is, the correct time. This puzzle will not be displayed directly. In the Gameplay view, you will see a Joystick to move the Player Model. The player can move the Camera vertically by touching the layers on the left and right, and will move in accordance with the direction of the logistics that it touches.

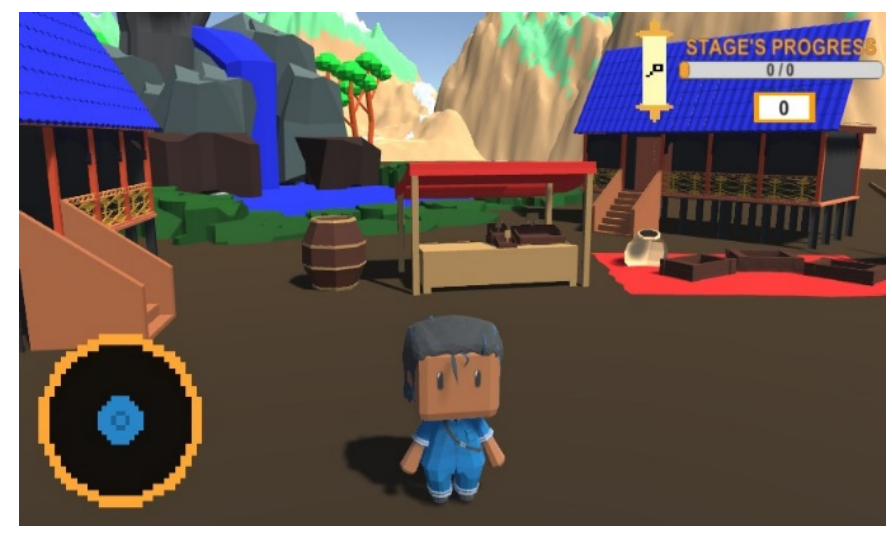

Figure 18: Gameplay Scene

Players will see a button in the form of Scoll or Scroll, which serves to display a Panel that contains instructions where the location of hidden questions.

Then, the Player must move to find it by exploring an area to find the point where the puzzle is located. HOTS games are linear which means the next puzzle will not appear before completing the previous one. 


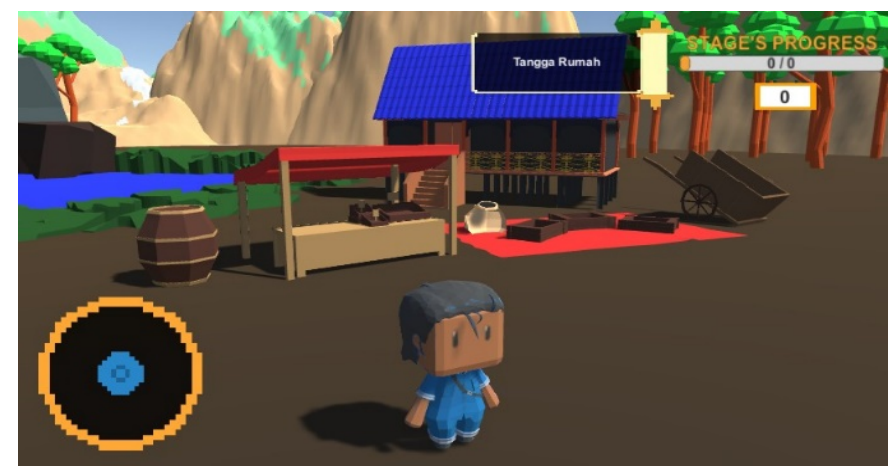

Figure 19: Gameplay showing the clue

After completing a set of puzzles in one area, the player gets hidden treasures in that area. Then the player will shown a UI or indication of a Task or Puzzle to complete.
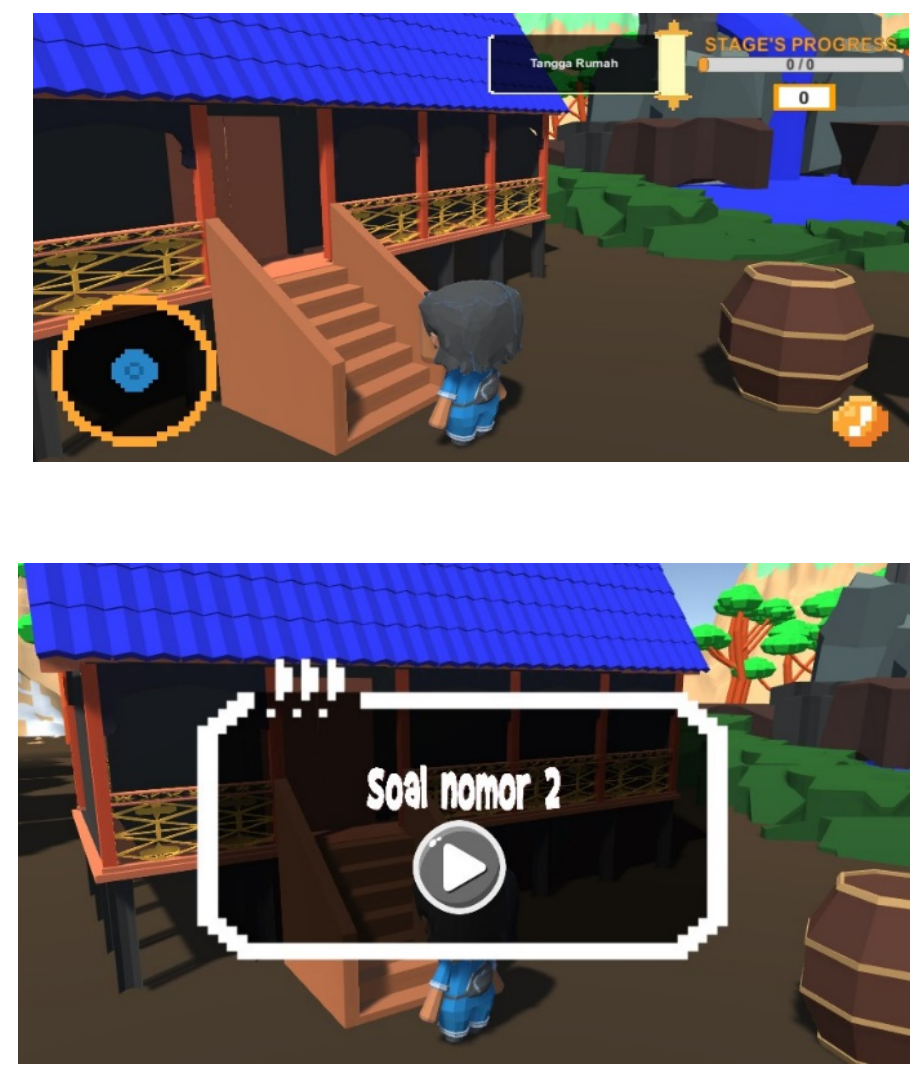

Figure 21: Appereance of Task no.2

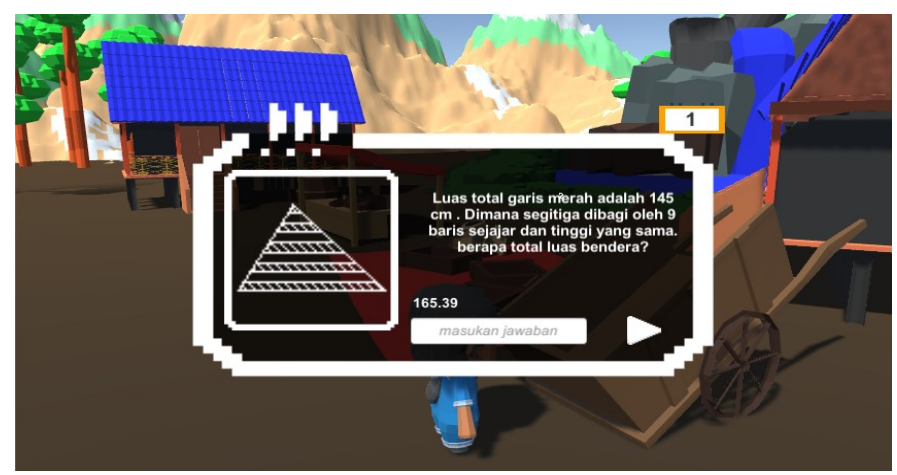

Figure 22: Task Display
To solve the puzzle in the form of mathematical problems, players are required to enter the correct number of digits after making calculations according to the existing problems. In addition to entering the correct numbers, players are required to interact with puzzles in the environment such as sliding, inserting, moving objects.

If the player answers correctly, the player will get a clue to the next puzzle and the player will get a score.

If the player answers incorrectly, the player will be given another chance to answer. If the second chance is still wrong, then the 'skip button' screen will appear to skip the puzzle problem and still be able to continue to the next puzzle.

The reward given is that the player will not get a score. In the exploration section, the gameplay display is third-person perspective. The player's character can explore the area in the game and can rotate the camera for different points of view.

Players must go around to find puzzles that have been marked and do interaction activities to start working on the puzzles found. When solving problems, in addition to getting instructions for the next question, there are questions that will get additional rewards, namely items that will be useful during gameplay exploration.

For example, a machete that will be used to cut branches to open roads covered with grass or branches. In each category, if you solve the problem until the last question, then you will get a reward in the form of a map of Indonesia. If it succeeds in collecting all the map fragments, it will display a map of Indonesia which shows famous points on Indonesia. For example, in the North Sumatra area there is the Lake Toba Icon, in the Yogyakarta Region there is the Borobudur Temple Icon.

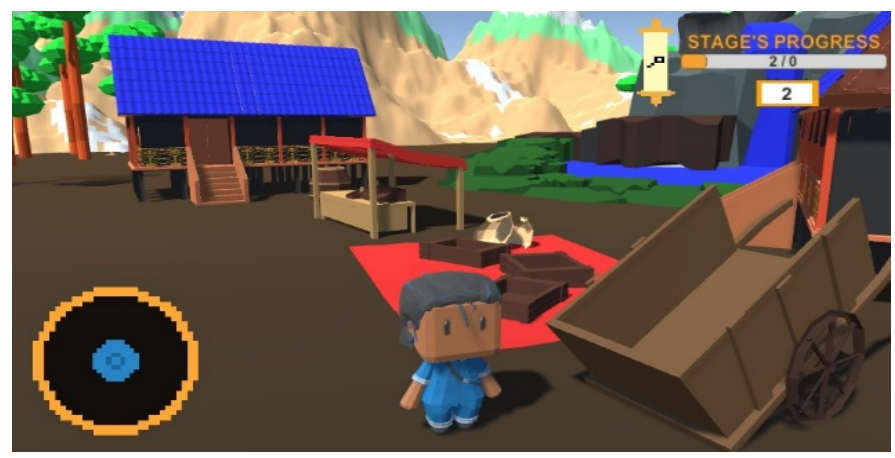

Figure 23: New Clue Appear

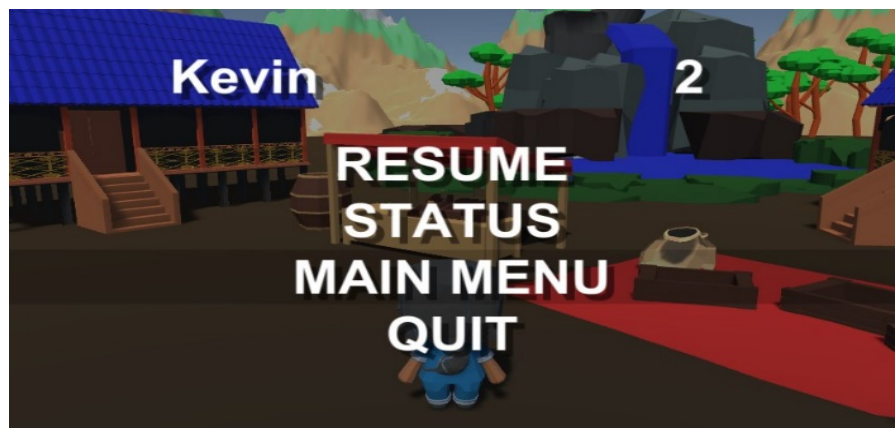

Figure 24: Display of Pause Menu 


\subsection{Pause Menu}

On the pause menu, there are several posts and buttons. There are two indications that show the username of the player and the score that has been obtained from the player.

Then there are several buttons. Namely Resume, Status, Main Menu, and Quit. On the Status menu, the player will be moved to a menu that shows details of player progressions when they play the game.

\subsection{Score}

Each question will give players 1 score and will accumulate on each Stage / island and will be shown to players via the pause menu and status menu. The status menu shows several indications, including the Score of the three Stages, the location of the player and the total score that has been obtained

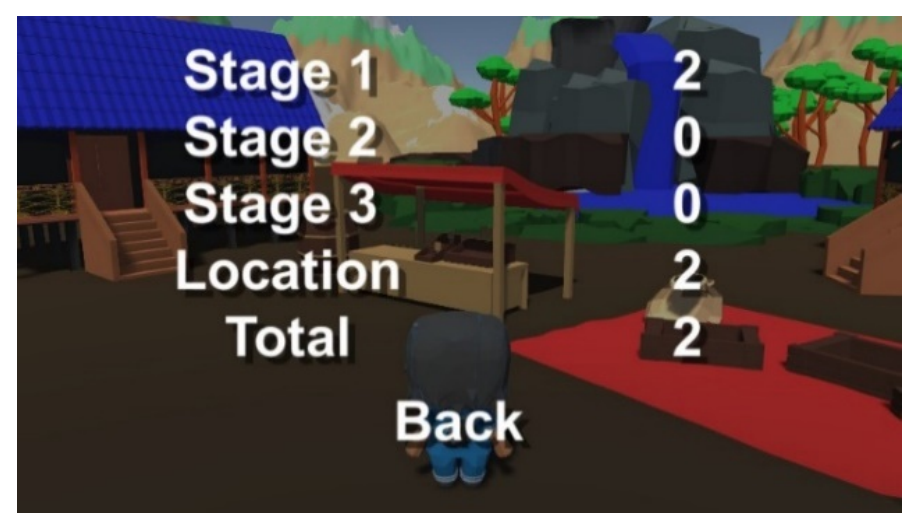

Figure 25: Display of Status Menu

\section{References}

[1] M.D. Aguilera, A. Mendiz, "Video games and education. Computers in Entertainment, 1(1), 1-10, 2003. https://doi.org/10.1145/950566.950583.

[2] Y. Udjaja, "Gamification Assisted Language Learning for Japanese Language Using Expert Point Cloud Recognizer", International Journal of Computer Games Technology, 2018. https://doi.org/10.1155/2018/9085179

[3] A.W. Ruch, "Videogame Interface: Artefacts and Tropes. Videogame Cultures and the Future of Interactive Entertainment, 2010. https://doi.org/10.1163/9781848880597_002

[4] M.D. Griffiths, "The educational benefits of videogames. Education and health, 20(3), 47-51, 2002.

[5] M. Hassenzahl, N. Tractinsky, "User experience - A research agenda. Behaviour and Information Technology, 25, 91-97, 2006. http://dx.doi.org/10.1080/01449290500330331

[6] D.P. Kristiadi et al., "The Effect Of Ui , Ux And Gx On Video", The IEEE CyberneticsCom 2017, 2017. https://doi.org/10.1109/CYBERNETICSCOM.2017.8311702

[7] P. Sporgis, "U.S. Patent No. 6,320,495. Washington, DC: U.S. Patent and Trademark Office, 2009

[8] M. Dondlinger, "Educational Video Game Design: A Review of the Literature. Journal of Applied Educational Technology, 4(1), 21-31, 2007.

[9] D.P. Kristiadi et al., "The effect of UI, UX and GX on video games", In 2017 IEEE International Conference on Cybernetics and Computational Intelligence (CyberneticsCom) 158-163, 2007. https://doi.org/10.1109/CYBERNETICSCOM.2017.8311702

[10] Sasmoko, J. Harsono, Y. Udjaja, Y. Indrianti, J. Moniaga,"The Effect of Game Experience from Counter-Strike" Global Offensive. In 2019 International Conference of Artificial Intelligence and Information $\begin{array}{llll}\text { Technology } & \text { (ICAIIT), } & 374-378, & \end{array}$ https://doi.org/10.1109/ICAIIT.2019.8834521

[11] Y. Udjaja, "Ekspanpixel Bladsy Stranica: Performance Efficiency Improvement of Making Front-End Website Using Computer Aided
Software Engineering Tool”, Procedia Computer Science, 135, 292-301, 2018. https://doi.org/10.1016/j.procs.2018.08.177

[12] Sasmoko, S.A. Halim, Y. Indrianti, Y. Udjaja, J. Moniaga,B.A. Makalew, "The Repercussions of Game Multiplayer Online Battle Arena. In 2019 International Conference of Artificial Intelligence and Information $\begin{array}{llll}\text { Technology } & \text { (ICAIIT), } & \text { 443-447, }\end{array}$ https://doi.org/10.1109/ICAIIT.2019.8834518

[13] Y. Udjaja et al., "Gamification for Elementary Mathematics Learning in Indonesia. International Journal of Electrical and Computer Engineering, 8(5), 3859, 2018. https://doi.org/10.11591/ijece.v8i5.pp38593865

[14] Y. Udjaja et al., "The Use of Role Playing Game for Japanese Language Learning", Procedia Computer Science, 157, 298-305, 2019. https://doi.org/10.1016/j.procs.2019.08.170

[15] Meiryani, Y. Udjaja, J. Jeviro, S. Sabrina, "The Utilization of Games in Learning Calculation (Accounting) Based on Andriod. International Journal of Psychosocial Rehabilitation, 24(9), 7-11, 2020. https://doi.org/10.37200/IJPR/V24I/PR290002

[16] I. Dart, M.J. Nelson, "Smart terrain causality chains for adventure-game puzzle generation", 2012 IEEE Conference on Computational Intelligence and Games, $\quad$ CIG, $328-334, \quad 2012$. https://doi.org/10.1109/CIG.2012.6374173. 